\title{
Macroscopic nucleon-nucleon correlations caused by the bounce-off process in energetic collisions of heavy nuclei
}

\author{
László P. Csernai* and Walter Greiner \\ Institut für Theoretische Physik, Johann Wolfgang Goethe Universität, \\ D-6000 Frankfurt am Main 1, Germany \\ Horst Stöcker, ${ }^{\dagger}$ Isao Tanihata, and Shoji Nagamiya \\ Lawrence Berkeley Laboratory, Berkeley, California 94720 \\ Jörn Knoll \\ Gesellschaft für Schwerionenforschung, D-6100 Darmstadt 11, Germany
}

(Received 3 December 1981)

\begin{abstract}
Two-particle correlation data are presented for the reaction $\mathrm{Ar}(800 \mathrm{MeV} /$ nucleon $)+\mathrm{Pb}$. The experimental results are analyzed in the nuclear fluid dynamical and in a linear cascade model. We demonstrate that the collective hydrodynamical correlations dominate the measured two-particle correlation function for the heavy system studied. We discuss the transition from the early stages of the reaction which are governed by few nucleon correlations, to the later stages with their macroscopic flow which can only be reached using heavy colliding systems. The sensitivity of the correlation data on the underlying compressional dissipative processes is analyzed.
\end{abstract}

$\left[\begin{array}{c}\text { NUCLEAR } \\ \text { REACTIONS Ar }+\mathrm{Pb}(800 \mathrm{MeV} / \text { nucleon }) \text { relativistic } \\ \text { heavy-ion reactions, two-proton correlations. }\end{array}\right]$

\section{INTRODUCTION}

We present an extension of a recent experimental study of two-particle correlations ${ }^{1}$ in relativistic heavy-ion reactions towards heavier collision systems. Also, theoretical aspects of the correlations $\mathrm{s}^{2,3}$ are discussed that cover two extremes of the reaction dynamics, namely the microscopic preequilibrium regime for which the linear cascade model is used and the collective macroscopic regime as accommodated by a fluid dynamical model.

Hydrodynamical models ${ }^{4,5}$ predict a preferred sideward emission of nuclear matter in central collisions of heavy nuclei as a result of the macroscopic matter flow caused by the strong pressure that builds up in the interaction zone. Such predictions are supported by the emission patterns of $\alpha$ particles and protons that have been observed in high multiplicity selected events of the particle track detector $^{5,6}$ and counter data, ${ }^{7,8}$ respectively. At intermediate impact parameters a different phenomenon is predicted in the hydrodynamical model $^{4,9}$ : The projectile matter as a whole essential- ly gets deflected by the target as a whole; the bounce-off effect. ${ }^{4,10}$ Cascade calculations, on the other hand, do not predict such an effect. The $180^{\circ}$ azimuthal correlation observed between light and heavy fragments ${ }^{8,13}$ has been the first experimental indication of this process. Here we want to investigate this phenomenon with an independent experiment, using nucleons as a probe. The latter have the distinct advantage that they do not significantly influence the overall balance of the conservation laws.

Studies with nucleon-nucleus $(n-N)$ collisions and with small nuclei showed that only limited thermalization may be reached in the light systems. ${ }^{1}$ The mean free path of an impinging proton in the nucleus was estimated ${ }^{14}$ to be approximately $2.4 \mathrm{fm}$. However, this value can be much smaller (around $\left.1.5 \mathrm{fm}^{14}\right)$ in nucleus-nucleus $(N-N)$ collisions due to the increasing temperature and density. Thus, while the lighter systems exhibit mainly the signatures of quasielastic nucleon-nucleon $(n-n)$ scattering, ${ }^{2}$ it is expected ${ }^{3}$ that in the heavier systems macrocorrelations such as the bounce-off process ${ }^{4}$ may dominate the observed correlations. 
The observation of nucleon-nucleon correlations that cannot be explained by quasifree $n-n$ scattering but require collective macroscopic flow of matter deserves particular interest. It is the aim of this paper to show that for the heavy systems studied the observed coincidence between two nucleons indicates the existence of such macroscopic correlations.

The paper is organized in the following way. We briefly recall the experimental setup in the next section. Subsequently we discuss the principle form of the two-particle coincidence cross section in the two dynamical regimes with reference to the two models employed. A discussion of the experimental results together with the theoretical predictions is given at the end.

\section{THE TWO-PARTICLE CORRELATION EXPERIMENT}

Two-particle correlations have been measured in the reaction $\mathrm{Ar}(800 \mathrm{MeV} /$ nucleon $)+\mathrm{Pb}$ at the
Berkeley Bevalac, completing the experimental study done previously ${ }^{1}$ for lighter systems. In this section we will not repeat all the details of the experimental setup; only basic features will be presented which are necessary for the further discussion. For more details see Refs. 1 and 15.

The experimental system consisted of a magnetic spectrometer $(S)$ and three sets of counter telescopes $(R, U$, and $D)$. These telescopes were set at scattering angles $(\theta, \Phi)=\left(40^{\circ}, 180^{\circ}\right), \quad\left(40^{\circ}, 90^{\circ}\right)$, and $\left(40^{\circ}, 270^{\circ}\right)$, respectively. The spectrometer $(S)$ was located at $\Phi=0^{\circ}$ and was rotated between $\theta=15^{\circ}$ and $110^{\circ}$. Although it was impossible to identify particles with the telescopes, it was known from the single particle inclusive data that the dominant yield at $\theta=40^{\circ}$ is protons. In the experiment presented here the telescope energy has been confined to $E_{\text {proton }} \geq 200 \mathrm{MeV}$.

An azimuthal (or coplanar) correlation function $C\left(y_{\|}, p_{T} / m\right)$ has been measured which is defined as

$$
C\left(y_{\|}, p_{T} / m\right)=\frac{2 \times\left[S\left(y_{\|}, P_{T} / m\right) \cdot R\right] / R}{\left[S\left(y_{\|}, P_{T} / m\right) \cdot U\right] / U+\left[S\left(y_{\|}, p_{T} / m\right) \cdot D\right] / D},
$$

where $y_{\|}$and $p_{T} / m=y_{T}$ are the rapidity coordinates of the particle detected by the spectrometer. The quantity $\left[S\left(y_{\|}, p_{T} / m\right) \cdot R\right]$ indicates the coincidence counts between the spectrometer and the $R$ telescope $\left(\Delta \Phi=180^{\circ}\right)$. The coincidence rates with the other two telescopes give the $\Delta \Phi=90^{\circ}$ correlations. At $\theta=0^{\circ}$ or $180^{\circ}\left(P_{T}=0\right)$ all these coincidence rates should be equal by definition, so that $C\left(y_{\|}, 0\right)=1$ there. It has a maximum $(C>1)$ at the rapidity where most of the inplane coincidences are observed. Out of plane correlations yield $C<1$.

The relative accuracy of the measured correlation function points is about $\Delta C=0.05-0.1$, but a slight difference between the sensitivities of the three telescopes could cause an overall normalization error of about $\Delta C=0.1-0.3$. This error could have been eliminated only by the measurement of $C\left(y_{\|}, 0\right)$ values, but the available polar angle range $\left(\theta=15^{\circ}-110^{\circ}\right)$ did not allow this.

\section{MICROSCOPIC TWO-PARTICLE CORRELATIONS AND THE TRANSITION FROM MICRODYNAMICS TO MACRODYNAMICS}

In this section we describe the principal theoretical form the two particle cross section attains in go- ing from the microdynamical regime towards the macroregime. At early times of the reaction only a few nucleons of both nuclei suffer only a few interactions. If one were to observe the nucleons at this early stage, the only strong correlations arise from such quasifree $n-n$ scattering contributions. Thus, the respective two-particle distribution function at a fixed impact parameter $b$ attains a form containing two terms ${ }^{16}$

$$
\begin{aligned}
{ }^{2} f\left(p_{1}, p_{2}, b\right)= & \left.\sum_{c}{ }^{2} f_{c}\left(p_{1}, p_{2}\right)\right|_{b} \\
& +\left.\sum_{c^{1} \neq c^{2}}{ }^{1} f_{c^{1}}\left(p_{1}\right){ }^{1} f_{c^{2}}\left(p_{2}\right)\right|_{b},
\end{aligned}
$$

where $c, c^{1}$, and $c^{2}$ denote all the possible subsystems (clusters) that were in interaction contact so far. ${ }^{17}$ Besides the possibility that both observed nucleons result from the same cluster $c$ with a correlated spectrum ${ }^{2} \mathrm{f}_{c}$, they can also result from two different clusters described by the uncorrelated product of the respective single particle momentum distributions ${ }^{1} f_{c}$. In the sense of the microregime discussed so far the latter term represents an uncorrelated background. During the later stages of the collision, however, this goes over into the macroscopic correlation term. By integrating over all 
impact parameters we obtain the two particle coincidence cross section:

$$
\begin{aligned}
d^{2} \sigma / d p_{1} d p_{2} & \equiv \sigma_{C}\left(p_{1}, p_{2}\right) \\
& =\int d^{2} b^{2} \mathbf{f}\left(p_{1}, p_{2}, b\right) .
\end{aligned}
$$

The microcorrelations prevail in light systems or at peripheral impact of heavy nuclei. However, in nonperipheral collisions of heavy nuclei the above clusters may attain a local (micro) equilibrium. Then the clusters turn into local fluid elements at position $r$ and the forthcoming interactions serve the relaxation on the macroscale, for which the fluid dynamical model may give an appropriate description. In the fluid dynamical model the clus- ters or infinitesimal fluid elements are assumed to be in local equilibrium. Therefore microscopic correlations do no longer appear; the second term in Eq. (2) becomes dominant. Then the two-particle spectrum has the form

$$
{ }^{2} F_{H}^{\text {cell }}\left(p_{1}, p_{2}, r\right)={ }^{1} F_{H}\left(p_{1}, r\right){ }^{1} F_{H}\left(p_{2}, r\right),
$$

where we use upper case letters to represent the macrodynamics and the subscript $H$ stands for hydrodynamics. In a collision at fixed impact parameter $b$ the total two-particle distribution contains the correlations arising from the collective flow: The neighboring cells are correlated to one another in the momentum space. The two-particle distribution is still separable:

$$
{ }^{2} F_{H}\left(p_{1}, p_{2}, b\right)=\left.\int d^{3} r_{1} d^{3} r_{2}{ }^{1} F_{H}\left(p_{1}, r_{1}\right)^{1} F_{H}\left(p_{2}, r_{2}\right)\right|_{b}={ }^{1} F_{H}\left(p_{1}, b\right)^{1} F_{H}\left(p_{2}, b\right) .
$$

The integration of Eq. (5) over all impact parameters $b, \int d^{2} b$ breaks this factorization and leads to the respective two-particle cross section

$$
{ }^{2} \sigma_{H}\left(p_{1}, p_{2}\right)=\int d^{2} b^{2} F_{H}\left(p_{1}, p_{1}, b\right) .
$$

Thus, observing one nucleon with momentum $p_{1}$ introduces a bias in the impact parameter, so that the second nucleon attains a spectrum other than the inclusive cross section (see also the Appendix and Fig. 5).

Hence, the cascade and hydrodynamical models both are describing correlations, but of different origin. In the preequilibrium regime, as adequately described by the cascade picture, the correlations between different clusters may be neglected, while the microscopic correlations are taken into account. On the contrary, in the hydrodynamical model microscopic correlations within one local cluster (fluid element) are neglected, while strong macroscopic correlations between different clusters (different regions) are considered.

It was one of the goals of the classical equation of motion approaches to study microscopically the transition from microdynamics to (collective) macrodynamics. In order to achieve a separation of these two regimes Bodmer et al., ${ }^{18}$ for instance, suggested dividing the $n-n$ force into long and short range parts: The long range part governing the macrodynamics in terms of an averaged force (Vlasov equation), the short range force entering the stochastic collision term. In this type of unified model there would be a possibility to study the gradual change of the correlation function in the transition region. In order to accommodate both the micro and the macro regimes in a more simple way, one might think of a phenomenological model that retains the microcorrelations from Eq. (2) and replaces its second (background) term by the respective hydrodynamical spectrum. Thus, integrating over all impact parameters, we obtain the coincidence cross section

${ }^{2} \sigma\left(p_{1}, p_{2}\right)=\int d^{2} b\left[{ }^{2} \mathbf{f}\left(p_{1}, p_{2}, b\right)+{ }^{2} F_{H}\left(p_{1}, p_{2}, b\right)\right]$.

This composition (7) physically means that we take into account the correlations arising in a single fluid cell due to the limited number of nucleons in the cell [first term of (7)]. The usual hydrodynamical descriptions neglect this effect. Such a unified model may enable the description of a smooth transition from the microregime prevailing in the early stage of the collision to the macroregime in the later stage.

The models actually employed for the two dynamical regimes are the linear cascade model and the hydrodynamical model, respectively. Compared to three-dimensional cascade calculations in the linear cascade model $^{2}$ special simplifying assumptions are made for the type and weights of the various possible contributions to (2). For further details we refer to Ref. 2, where a systematic study of the correlations in light systems is also given. The hydrodynamic model ${ }^{4}$ that describes the violent stage 
of the reaction following the preequilibrium regime is supplemented by an evaporation calculation. ${ }^{19}$ The latter describes the transition from the fluid phase (hot nuclear matter) to separate nucleons and nuclei by a sudden breakup at the late stage of the expansion. Since this calculation includes the production of composite particles, only a fraction of the nucleons leave the interaction zone as free nucleons. A detailed description of the fluid dynamical model with evaporation is given in Refs. 19 and 20, and summarized in the Appendix.

\section{RESULTS AND DISCUSSION}

Let us first recall the kinematical situation of the experiment. Figure 1 explains the circumstances in the rapidity plane. The hatched area indicates the sensitivity range of the telescopes, while for the inplane coincidence, the spectrometer maps out the lower part of the rapidity plane. By intent, the chosen angle and energy cuts of the telescopes favor an analysis of the quasifree scattering process (knockout). It leads to rapidities close to the dashed kinematical curve (a circle in nonrelativistic kinematics) with a near back-to-back emission relative to the $n-n$ c.m. frame. The isolation of this process, which in fact leads to the most pronounced microcorrelation, was one of the major motivations for the present experimental setup.

In fact, earlier coincidence experiments on light systems ${ }^{1}$ showed an enhancement of the in-plane coincidence yield, in line with the expectations from the quasifree scattering process. A detailed discussion of these data by a phase space model ${ }^{16}$ and the simplified cascade picture ${ }^{2}$ further showed that besides the knockout part, there is also a sizable correlation among the cluster nucleons due to the common share of energy and momentum, if mainly small clusters contribute. The latter effect gives rise to an in-plane correlation dominating at large momenta. ${ }^{2}$

The above described effects are evidently not the ones that we see in the coincidence rates of the present experiment, as shown in Figs. 2(a) and (c). Rather, for this heavy collision system we observe a disfavor of the in-plane coincidence rate at large spectrometer momenta along with an in-plane enhancement at moderate momenta (with respect to the target frame). The correlation function $C$ [Eq. (1)] calculated in the framework of the linear cascade model in fact shows a qualitative disagreement with the experimental result. In this cascade model

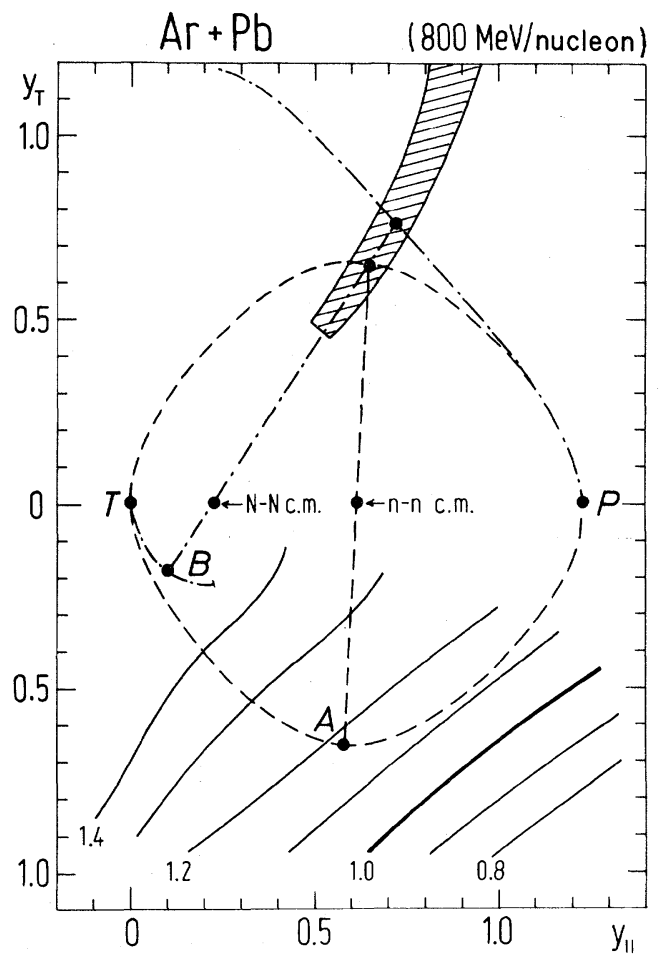

FIG. 1. Contour plot of correlation function $C$ [Eq. (1)] for $\mathrm{Ar}+\mathrm{Pb}$ at $800 \mathrm{MeV} /$ nucleon projectile energy. $P$ and $T$ indicate projectile and target momenta per nucleon, respectively, in the nucleon-nucleon $(n-n)$ c.m. frame. The dashed circle indicates the free proton elastic scattering kinematics, the cross hatched area shows the kinematical region of protons detected by the $R$ telescope, and the point $A$ the expected position of the quasielastic $n-n$ knockout peak. The dashed-dotted circles indicate the collective nucleus-nucleus $(N-N)$ scattering kinematics. The maximum is obtained experimentally where it is expected in the collective $N$ - $N$ kinematics $(B)$.

an enhancement is obtained around the quasielastic $n-n$ peak, as in lighter systems. However, here this increase is rather weak, $C \simeq 1.1$. This points towards the expectation that in large systems the background term [second term in (2)] becomes so large that the correlation part of the model [first term in (2)] reaches the $15 \%$ level of the total coincidence yield at most. The fact that the obtained $C$ function does not resemble the experimental one shows the influence of effects other than microscopic two- or few-particle correlations. How can we understand the structural change in going from the light to the heavy systems?

What are the effects that are left out in the sim- 


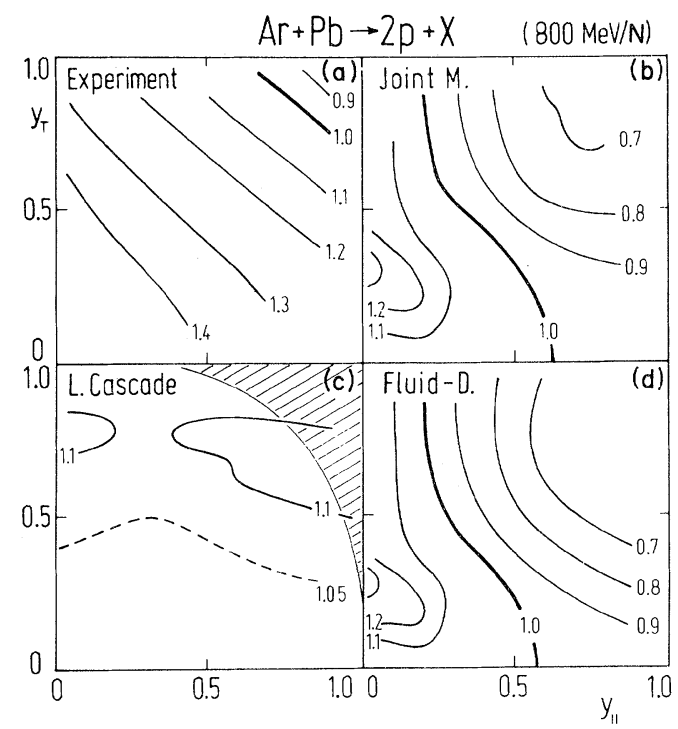

FIG. 2. (a) Contour plot of the correlation function $C$ measured in the reaction $\operatorname{Ar}(800 \mathrm{MeV} /$ nucleon $)+\mathrm{Pb}$ and shown in the rapidity plane. The relatively high maximum $(C \geq 1.4)$ in the vicinity of the target rapidity means that strong inplane correlation is found, but not at the point where it is expected on the basis of nucleonnucleon quasielastic scattering. (b) Contour plot of the correlation function $C$ for the same reaction calculated in the joint hydrodynamical and linear cascade model. The difference in the normalization might be due to the normalization error $\Delta C \simeq 0.1-0.3$ of the experiment (see Sec. II). (c) Contour plot of the correlation function $C$ for the same reaction calculated in the linear cascade model. Statistical fluctuations are eliminated by Gaussian smoothing. At the high rapidity values (hatched area) the statistical error is $\Delta C \geq 0.2$. (d) Contour plot of the correlation function $C$ for the same reaction calculated in the hydrodynamical model with a simple parabolic equation of state (Refs. 4 and 20) $(K=200 \mathrm{MeV})$.

plified cascade approach? The different clusters of interacting nucleons are treated as independent in this model. Thus, the contact of the clusters with the surrounding matter is neglected. This has essentially two effects: First, nucleons emitted from a certain cluster may rescatter. This leads to shadowing effects that distort the predicted correlations. Secondly, as already discussed in Sec. III, the forthcoming interactions serve a transport of energy and momentum across the different clusters and one enters the regime of macromotion. Thus, the data may draw attention towards collective effects.

First let us recall the earlier discussions of sha- dowing effects. ${ }^{1,15,21}$ The shadowing (rescattering) by the spectator nuclei suppresses the in plane coincidence rate as compared to the out of plane coincidence rate. We therefore expect $C<1$. A simple calculation based on the geometry and mean free path, however, can only reproduce the data at high forward momenta due to this shadowing effect. It fails completely at large angles where the value of $C$ is larger than 1. We therefore have to look for a different mechanism.

Returning to the kinematical situation depicted in Fig. 1, if the projectile and the target act as a whole with their total inertias, then (ignoring inelasticity effects for the moment) one expects nucleons resulting from the decay of the deflected projectile and target fragments to occupy the kinematical regimes around the dashed-dotted lines. This is essentially the physics of the bounce-off $\mathrm{f}^{4}$ process seen in hydrodynamical calculations. As it was shown qualitatively in the bounce-off model, ${ }^{3}$ this process may lead to a correlation function similar to the experimental one. Now in a detailed three dimensional hydrodynamical and evaporational ${ }^{4,22}$ model the triple differential proton cross section is evaluated (Fig. 3) and the two-particle correlation function is

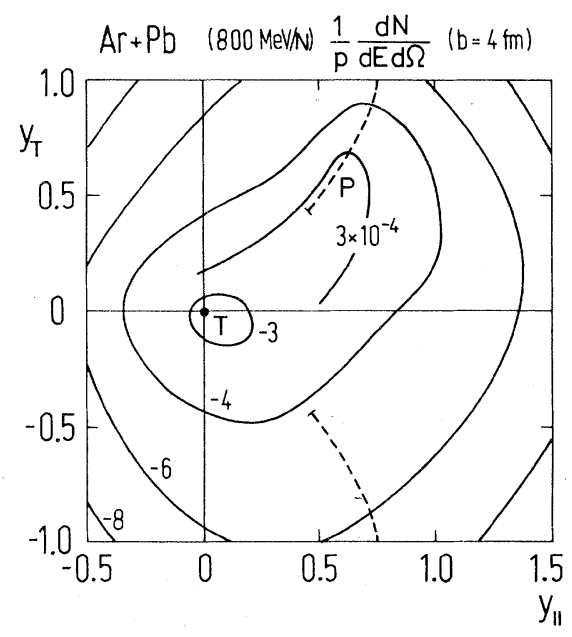

FIG. 3. Contour plot of the triple differential invariant cross section $(1 / p) d^{3} N / d E d \Phi d \cos \theta$ for the reaction $\mathrm{Ar}(800 \mathrm{MeV} /$ nucleon $)+\mathrm{Pb}$ at impact parameter $b=4 \mathrm{fm}$ in the reaction plane $\left(\Phi=0^{\circ} / 180^{\circ}\right)$ calculated in the hydrodynamical model. The contour lines labeled by parameter $q$ are corresponding to a value of $10^{q} /(\mathrm{sr}$ $\mathrm{MeV}^{2}$ ). The dashed lines indicate the kinematical region where protons are detected by the counter telescopes $R, U$, and $D$ in the experiment described above (see Ref. 1 and Chap. II). The points $T$ and $P$ show the target and projectile evaporation peaks. The telescopes are predominantly sensitive for projectile evaporation according to the model. 
calculated on the basis of Eqs. (1) and (6) (see also the Appendix).

The coincidence function is in good qualitative agreement with the experimental observations [Fig. 2(d)]. The maximum around the zero rapidity is caused by the target evaporation which anticorrelates azimuthally with the higher energy projectile evaporation detected by the telescopes. The position of the maximum agrees with the experimental observations. The maximum of $C$ is somewhat lower $(\simeq 0.2)$ than in the experiment but this difference is within the systematic experimental normalization error. The difference between the maximum and minimum values is only slightly higher than in the experiment. This shows that additional effects may influence the coincidence function, and the structure of the $C$ function should be smoother by $10-20 \%$. In the framework of the fluid dynamical model, an extremely soft equation of state (phase transition) could cause stronger dissipation and so a stronger thermal smearing. However, finite particle number effects, i.e., nonthermal fluctuations and the microscopic nucleon-nucleon correlations $^{2}$ or quantum mechanical correlations, ${ }^{21}$ can cause similar smearing effects.

If we assume that the local nucleon clusters within the nuclear fluid have sizes and momentum distribution similar to the ones predicted by the linear cascade model, we can evaluate the modifying effects arising from the microscopic correlations. The $C$ function obtained in a joint hydrodynamical and cascade model [Eq. (7)] resembles mainly the features of the hydrodynamical model [Fig. 2(b)]; now the experimentally observed slope between the maximum and minimum is also reproduced. This shows that the consideration of the finite nucleon cluster effects within the nuclear fluid may extend the validity of such a unified model to the intermediate mass regions where neither the cascade nor the hydrodynamical model is sufficiently accurate.

\section{CONCLUSIONS}

It is important to note what type of underlying physical effects can be studied in this experiment in heavy $N-N$ systems. As it was shown in Fig. 1, the two-particle correlations measure the $N-N$ kinematics. As we have seen, the crucial point in the experiment is the lower energy cutoff of the telescopes. Owing to the $200 \mathrm{MeV}$ cutoff, the probability of detecting a proton from the target evaporation is two orders of magnitude smaller than is that from the projectile evaporation (Fig. 3). At the cutoff energy where both the target and the projectile evaporations can be detected by the telescopes equally, the collective character of the coincidence function vanishes because the collective azimuthal correlation cannot be exploited. By changing the lower energy cutoff of the telescopes and their $\theta$ angle, the bounce-off process could be mapped at different deflection angles. The position of the projectile evaporation peak could be estimated and thus the inelasticity could be measured, as shown in Fig. 4. On the other hand, the deflection angle and inelasticity and their dependence on the equation of state and viscous coefficients can be tested in hydrodynamical models. ${ }^{4,19,20}$ So this experiment provides an alternative tool for the investigation of the collective processes.

Another important point is to approach the reaction mechanism by this type of study. We have seen two types of correlations show up in the discussed experiment. These represent two different stages on the way towards the equilibration. The way to local thermo- and fluid-dynamical equilibrium leads through the buildup of small interacting clusters first and then later these clusters may grow. The initially unimportant weaker interactions between the clusters later become more important and at later stages the signs of this collective type of

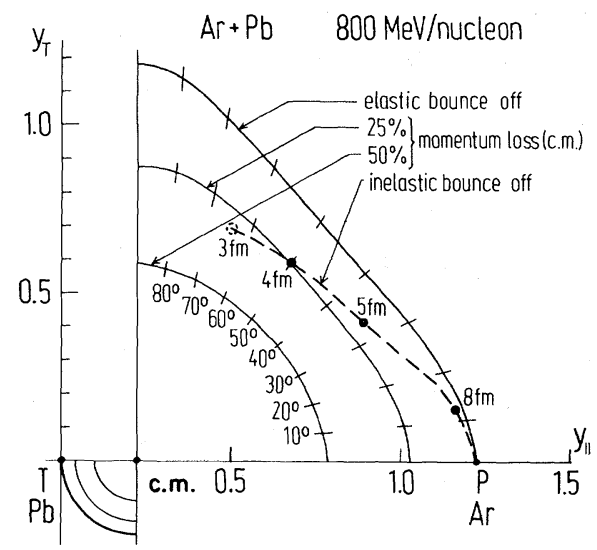

FIG. 4. The dependence of the c.m. bounce off deflection angle and inelasticity on the impact parameter $b$. At the impact parameter $b=4 \mathrm{fm}$, the bounce off angle is $\theta \simeq 48^{\circ}$ and $25 \%$ for the c.m. collective momentum is lost. At impact parameters lower than $3 \mathrm{fm}$ the second local maximum of the spectrum vanishes and the inelasticity cannot be uniquely determined, but the bounce off angle is measurable. 
correlations show up. In very small systems the reaction cannot reach this stage. ${ }^{23} \mathrm{~A}$ major part of nucleons leave the system before larger clusters and collective processes may develop. Otherwise, in larger systems the amount of nucleons escaping from initially independent clusters becomes negligible and mainly the signs of collective correlations can be observed. When already collective processes start to develop, the reaction mechanism may be influenced considerably by especially strong long range correlations ${ }^{21}$ caused by phase transitions, for example. $^{24}$ By a systematic study of the microscopic and macroscopic correlations, the collective processes may be separated from other effects and then their properties and anomalies may indicate to us the signs of the extreme states occurring in hot and dense nuclear systems.

The present results are promising and on this basis we hope that the present experimental and theoretical investigations to test the triple differential nucleon correlation cross section will provide us with accurate quantitative information about the properties of nuclear matter and the reaction mechanism.

\section{ACKNOWLEDGMENTS}

The authors thank Miklós Gyulassy for suggesting the present theoretical analysis. This work was supported by the Alexander von Humboldt Foundation, the Deutscher Akademischer Austauschdienst, the Bundesministerium für Forschung und Technologie, and by the U. S. Department of Energy under Contract W-7405-ENG-48.

\section{APPENDIX}

Here we briefly present the evaporational model and the calculation of the correlation function based on it.

At the breakup moment in each point $r$ the fluid is moving with the local collective velocity $v(r),\left[\beta_{r}=v(r) / c\right.$, in the following the $c \equiv 1$ convention will be used]. The thermally equilibrated nucleon distributions $\mathrm{f}(p, r)$ [normalized to the particle densities as $\left.n(r)=\int d^{3} p f(p, r)\right]$ should be transformed to the laboratory system by a Lorentz transformation from each fluid cell:

$$
\begin{aligned}
{ }^{1} F_{H}(\boldsymbol{P}, \boldsymbol{r}) & ={ }^{1} \boldsymbol{F}_{H}^{\mathrm{lab}}(\boldsymbol{P}, \boldsymbol{r}) \\
& =\left[w_{r}(\boldsymbol{P}, \boldsymbol{W}) / \boldsymbol{W}\right] f^{\mathrm{cell}}\left(p_{r}(\boldsymbol{P}, W), r\right),
\end{aligned}
$$

where $p_{r}, w_{r}(\boldsymbol{P}, \boldsymbol{W})$ are rest frame (laboratory) four-

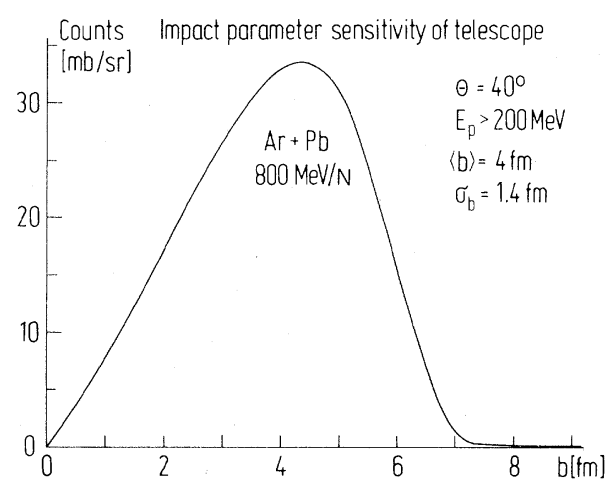

FIG. 5. The impact parameter dependence of the sensitivity of telescopes $R(b)$ [ $U(b)$ and $D(b)$ ] in the hydrodynamical model [as given in Eq. (12)].

momenta, respectively. (The experimental observables are $P$ and $W$, the corresponding cell fourmomenta are depending not only on $P$ and $W$, but also on $r$.) They are connected by the relations:

$$
\begin{aligned}
& w_{r}(P, W)=\gamma_{r}\left(W-\beta_{r} P\right), \\
& p_{r}(P, W)=P-\gamma_{r} \beta_{r}\left(w_{r}+W\right) /\left(1+\gamma_{r}\right), \\
& \gamma_{r}=1 / \sqrt{1-\beta_{r}^{2}} .
\end{aligned}
$$

The local momentum distributions $f^{\text {cell }}(p, r)$ in the rest frame of the matter were approximated in the following way: As in Refs. 4 and 20, only the free nucleons were taken into account. For these a relativistic Fermi-Dirac momentum distribution $f^{\text {cell }}(p, r)$ was applied. This was then shifted down in energy by the local binding (obtained at the breakup from the long range potentials and the equation of state used in the fluid dynamical model) and only those nucleons were allowed to evaporate which had positive energy in this distribution $(15-40 \%)$. The differential cross section

$$
d \sigma / d P \equiv{ }^{1} \sigma(P)=\int d^{2} b d^{3} r^{1} F_{H}(P, r)
$$

is obtained by adding the contributions of all fluid cells $i$ in the laboratory system ${ }^{19}$ and then summing up the results of the different impact parameter calculations weighted by the corresponding geometrical surfaces. Changing the variables of the cross section from the momentum to energy and angles we write the triple-differential cross section at a fixed impact parameter as: 


$$
d^{3} N / d E d \Phi d \cos \theta \equiv{ }^{1} \omega(\theta, \Phi, E, b)=\sum_{i} \operatorname{Vol}_{i} w_{i}(P, W) \sqrt{W^{2}-m^{2}} f_{i}^{\text {cell }}\left(p_{i}(P, W)\right),
$$

where,

$$
{ }^{1} \omega={ }^{1} \sigma(P)\left[d^{3} P / d E d \Phi d \cos \theta\right]={ }^{1} \sigma(P(E, \theta, \Phi)) W / \sqrt{W^{2}-m^{2}}, \quad E=W-m,
$$

the spatial integration in Eq. (A3) is replaced by the sum over the fluid cells $i$ having the volume $\mathrm{Vol}_{i}$, and the Lorentz transformation is applied [Eqs. (A1) and (A2)]. The triple differential cross section obtained with normal equation of state ${ }^{4}$ (i. e., without phase transition) provides a peak in the impact parameter sensitivity of the telescopes $R, U$, and $D$ at $b=4 \pm 1.4 \mathrm{fm}$ (Fig. 5),

$$
R(=U=D)=\int R^{\prime}(b) d^{2} b=\int{ }^{1} \omega\left(\theta=40^{\circ}, \Phi, \epsilon \geq 200 \mathrm{MeV}, b\right) d \Phi d \epsilon d^{2} b
$$

and this peak is about two times as sharp as would be expected in the fireball model. ${ }^{25}$ At this impact parameter the deflection angle is $\theta \simeq 48^{\circ}$ in the c.m. system and $\sim 25 \%$ of the collective projectile and target momenta are lost. According to Eqs. (5) and (6) and considering the experimental restrictions, we can evaluate the coincidence rate between the telescope $R(U, D)$ and spectrometer $S, \delta=180^{\circ}$ (in the case of $U$ and $D$ telescopes $\delta=90^{\circ}$ and $270^{\circ}$, respectively):

$$
\left[S\left(y_{\|}, y_{T}\right) \cdot R\right]=\int{ }^{1} \omega\left(\theta^{2}=40^{\circ}, \Phi^{2}, \epsilon^{2} \geq 200 \mathrm{MeV}, b\right){ }^{1} \omega\left(y_{\|}, y_{T}, \Phi^{2}+\delta, b\right) d \Phi^{2} d \epsilon^{2} d^{2} b .
$$

Using the quantities [(A5) and (A6)] the correlation function $C$ can be evaluated by Eq. (1).

"Present address: Central Research Institute for Physics, 1525 Budapest, Hungary.

${ }^{\dagger}$ Present address: Gesellschaft für Schwerionenforschung, D-6100 Darmstadt 11, Germany.

${ }^{1}$ S. Nagamiya, L. A. Anderson, W. Bruckner, O. Chamberlain, M. C. Lemaire, S. Schnetzer, G. Shapiro, H. Steinber, and I. Tanihata, Phys. Lett. $\underline{81 B}, 147$ (1979); I. Tanihata, M. -C. Lemaire, S. Nagamiya, and S. Schnetzer, ibid. 97B, 363 (1980).

2J. Knoll, and J. Randrup, Phys. Lett. 103B, 264 (1981).

${ }^{3}$ L. P. Csernai and W. Greiner, Phys. Lett. 99B, 85 (1981).

${ }^{4}$ H. Stöcker, J. Maruhn, and W. Greiner, Phys. Rev. Lett. 44, 725 (1980); Z. Phys. A 293, 173 (1979); H. Stöcker, L. P. Csernai, G. Graebner, G. Buchwald, H. Kruse, R. Y. Cusson, J. A. Maruhn, and W. Greiner, Phys. Rev. C (to be published); H. Stöcker et al., Phys Rev. Lett. 47, 1807 (1981).

${ }^{5}$ H. G. Baumgardt, J. U. Schott, Y. Sakamoto, E. Schopper, H. Stöcker, J. Hofmann, W. Scheid, and W. Greiner, Z. Phys. A 273, 359 (1975); J. Hofmann, H. Stöcker, U. Heinz, W. Scheid, and W. Greiner, Phys. Rev. Lett. $\underline{36}, 88$ (1976).

${ }^{6}$ H. G. Baumgardt and E. Schopper, J. Phys. (London) Lett. G $\underline{5}$, L231 (1979).

${ }^{7}$ R. Stock, H. H. Gutbrod, W. G. Meyer, A. M. Poskanzer, A. Sandoval, J. Gosset, C. H. King, G. King, Ch. Lukner, Nguyen Van Sen, G. D. Westfall, and K. L. Wolf, Phys. Rev. Lett. 44, 1243 (1980).

${ }^{8}$ H. H. Gutbrod, Lawrence Berkeley Laboratory Report
No. LBL-11123, 1980; Proceedings of the Joint JapanU. S. Seminar on High Energy Nuclear Interactions, Hakone, Japan 1980, edited by K. Nakai and A. S. Goldhaber, p. 93.

${ }^{9}$ A. A. Amsden, G. F. Bertsch, F. H. Harlow, and J. R. Nix, Phys. Rev. Lett. 35, 905 (1975); A. A. Amsden, F. H. Harlow, and J. R. Nix, Phys. Rev. C 15, 2059 (1977); A. A. Amsden, A. S. Goldhaber, F. H. Harlow, and J. R. Nix, ibid. 17, 2080 (1978).

${ }^{10}$ Spherical (see Ref. 11) and axial symmetric (see Ref. 12) models are not able to predict this phenomenon.

11J. P. Bondorf, S. Garpmann, and J. Zimányi, Nucl. Phys. A296, 320 (1978); and L. P. Csernai, B. Lukács, and J. Zimányi, Lett. Nuovo Cimento 27, 111 (1980).

12P. Danielewicz, Nucl. Phys. A314, 465 (1979); H. H. Tang and C. Y. Wong, Phys. Rev. C 21, 1846 (1980).

${ }^{13}$ W. G. Meyer, H. H. Gutbrod, Ch. Lukner, and A. Sandoval, Phys. Rev. C 22, 179 (1980).

${ }^{14}$ I. Tanihata, S. Nagamiya, S. Schnetzer, and H. Steiner, Phys. Lett. 100B, 121 (1981); J. D. Stevenson, J. Martins, and P. B. Price, Phys. Rev. Lett. 47, 990 (1981).

${ }^{15}$ I. Tanihata, Lawrence Berkeley Laboratory Report No. LBL-11320, 1980.

16J. Knoll, Phys. Rev. C 20, 773 (1979); Nucl. Phys. $\underline{\text { A343, }} 511$ (1980); J. Knoll and J. Randrup, ibid. A324, 445 (1979).

${ }^{17}$ J. Cugnon, T. Mizutani, and J. Vermeulen, Nucl. Phys. $\underline{\text { A352, }} 505$ (1981); J. Cugnon and S. E. Koonin, ibid. A355, 477 (1981); J. Cugnon, J. Knoll, and J. Randrup, ibid. $\underline{\text { A360, }} 444$ (1981). 
${ }^{18}$ A. R. Bodmer, C. N. Panos, and A. D. MacKellar, Phys. Rev. C 22, 1025 (1980).

${ }^{19}$ L. P. Csernai and H. W. Barz, Z. Phys. A 296, 173 (1980).

${ }^{20} \mathrm{G}$. Buchwald, L. P. Csernai, J. Maruhn, W. Greiner, and H. Stöcker, Phys. Rev. C 24, 135 (1981).

${ }^{21}$ M. Gyulassy, Lawrence Berkeley Laboratory Report No. LBL-12389, 1981.

${ }^{22}$ Without the thermal evaporation the flow velocity dis- tribution alone would yield too strong a correlation.

${ }^{23}$ H. W. Barz, L. P. Csernai, and W. Greiner, Phys. Rev. $\mathrm{C}$ (to be published).

${ }^{24}$ M. Gyulassy, and W. Greiner, Ann. Phys. (N.Y.) 109, 485 (1977).

25J. Gosset, H. H. Gutbrod, W. G. Meyer, A. M. Poskanzer, A. Sandoval, R. Stock, and G. Westfall, Phys. Rev. C 16, 629 (1977). 